\title{
Diferencias sociodemográficas en la satisfacción marital: el caso de México ${ }^{1}$
}

\author{
SUSAN PICK DE WEISS y PATRICIA ANDRADE PALOS \\ Facultad de Psicología \\ Universidad Nacional Autónoma de México e Instituto Mexicano \\ de Investigación de Familia y Población
}

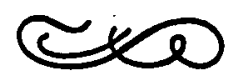

\section{Resumen}

Este estudio se refiere a un anălisis de las diferencias sociodemográficas que estän presentes en la satisfacción marital en relación a la interacción en las parejas, aspectos emocionales y estructura. les del cónyuge. Las variables sociodemográficas que se consideran son, a saber: sexo, número de años de casados, edad, escolaridad y número de hijos.

Se aplicaron las tres subescalas de la Escala de Satisfacción Marital (Pick de Weiss y Andrade Pa. los, sometido a publicación) en la Ciudad de México, a una muestra de 244 personas casadas.

Se encontraron diferencias con respecto al sexo del sujeto, su escolaridad y número de años casados, en lo que respecta a la satisfacción con la interacción conyugal. En relación a satisfacción con aspectos emocionales del cónyuge se obtuvieron diferencias en el nümero de hijos y las variables que mostraron diferencias significativas en el factor de satisfacción con aspectos estructurales del cónyuge fueron sexo y nümero de bijos.

\section{Abstract}

This sruay rejers to an anaiysts of the sociodemographic differences that are present in marital satisfaction in relation to couple interaction, emotional and structural aspects of the marital partner. The sociodemographic variables that are considered are sex, number of years of marriage, age, schooling and number of children.

The three subscales of the Marital Satisfaction Scale (Escala de Satisfaccion Marital, ESM) (Pick de Weiis y Andrade Palos, submited for publication), were applied in Mexico City to a sample of 244 married subjects.

Differences were found with respect to sex of the respondent, schooling and number of years married and satisfaction with the marital interaction. In relation to satisfaction with emotional aspects of the couple, differences were obtained regarding the number of children and lastly, the variables which showed significant differences in the factor dealing with structural aspects of the marniage partner were sex and number of offspring.

1 Ponencia presentada en el Primer Congreso Mexicano de Psicología Social, octubre, 1986

Direcciōn del autor: Universidad Nacional Autónoma de México. Facultad de Psicología. México D. F. 
La satisfacción marital ha sido definida por un lado, en relación a la evaluación que se hace de la pareja (Blood y Wolfe, 1960, Hicks y Platt, 1970) y por otra parte basándose en la evaluación de aspectos específicos de la vida marital (Burr, 1970; Chadwick, Albrecht y Kunz, 1976). Con el objeto de tener un instrumento válido y confiable para poblaciones en América Latina, específicamente México, recientemente fue desarrollada y validada la Escala de Satisfacción Marital (Pick de Weiss y Andrade Palos, sometida a publicación).

La satisfacción marital en la literatura ha sido medida fundamentándose en dos tipos de definiciones: una en términos de ajuste en la pareja (Locke y Wallace, 1959, Spanier, 1976) y otra con respecto a las actitudes que prevalecen hacia la relación marital (Roach, Frazier y Bowden, 1981). En el desarrollo de la Escala de Satisfacción Marital (ESM) se tiene como base esta última definición, ya que su objetivo es el de tener más forma de medir cambios en la percepción o en la posición del individuo más que una evaluación del estado del matrimonio en sí.

Berget y Kellner (1970) expresan claramente esta posición al sugerir que no es lo que sucede en el matrimonio sino cómo lo definen los miembros, lo que es de relevancia para la comprensión y estudio de la relación marital. La satisfacción marital es entonces una realidad construida por los miembros de la diada marital.

Como se puede observar en revisiones bibliográficas sobre satisfacción marital (Hicks y Platt, 1970; Laws, 1971) existe un gran número de investigaciones en esta área, pero ninguna con latinoamericanos utilizando un instrumento diseñado específicamente para estos grupos, de ahí la necesidad de realizar estudios de estas culturas.

El objetivo de la presente investigación es analizar la satisfacción marital y las diferencias que existen en ella, de acuerdo a variables tales como sexo, número de años del matrimonio, edad, nivel educativo y número de hijos en una muestra de personas casadas que habitan en la Ciudad de México.

En lo que se refiere a diferencias en la satisfacción marital entre hombres y mujeres, existen resultados contradictorios. Algunos autores muestran diferencias (Atkinson, 1980; Campbell y cols. 1976; Hicks y Platt, 1970; Rollins y Feldman, 1970) y otros no (Rollins y Cannon, 1974). Además, aunque Rollins y Feldman (1970) encuentran que hay diferencias, en un análisis posterior de los mismos datos (Rollins y Cannon, 1974), se confirma que no las hay, de donde los autores concluyen que los cónyuges de ambos sexos se ven influidos de manera similar en lo que respecta a la satisfacción marital por eventos que ocurren en diferentes etapas del ciclo vital.

Más recientemente, Rollins y Galligan (1978) encontraron que las mujeres se ven más afectadas en su satisfacción marital por la edad y la presencia de niños, que los hombres. Rhyne (1981) propone que aunque los hombres muestran niveles más altos de satisfacción marital, son los mismos factores los que determinan el grado de satisfacción marital en hombres y mujeres y que la importancia de estos factores varía de una etapa a otra.

Una de las variables más ampliamente estudiada en otras culturas en relación a la satisfacción marital, es el ciclo de la pareja, medida ya sea en términos de número de años de matrimonio o de la edad de cada uno de los miembros.

En relación al número de años de matrimonio se ha visto que la satisfacción es mayor en el período inicial y final de la pareja y menor en el período intermedio (Burr, 1970; Rollins y Feldman, 1970; Rollins y Cannon, 1974). Algunas 
explicaciones que se han postulado respecto a esta relación son la presencia de hijos y el cambio de roles familiares que se tienen en diferentes etapas del matrimonio.

Otros autores han encontrado un decremento lineal en la satisfacción desde el inicio de la relación hasta los años que se acercan al final de ésta (Pineo, 1961; Swensen, Eskew y Kohlepp, 1981).

A pesar de la relevancia que se le ha dado en la literatura al ciclo vital, medido ya sea en términos de edad de los cónyuges (Baltes, 1968), número de años de casados (Nock, 1979; Spanier, Sauer y Larzclere, 1979) o edad del hijo mayor (Duvall, 1971), se ha encontrado que esta variable aunque es un correlato significativo de la satisfacción marital, sólo explica un pequeño porcentaje de la varianza de ésta (Rollins y Cannon, 1974). Al respecto Baltes (1968) sugiere que los cambios en el ajuste marital en diferentes etapas, son simplemente función de la edad de los cónyuges y de percepciones relacionadas con ésta y no de los cambios en la relación conyugal.

Spanier, Lewis y Cole (1975) tras haber llevado a cabo una revisión de estudios en el área de ajuste y satisfacción marital, concluyen que la mayoría de ellos concuerdan en que inicialmente existe un decremento en la satisfacción de la pareja o en el ajuste marital, pero que, la velocidad y la intensidad de la reducción varía de un estudio a otro.

En relación al nivel de escolaridad, Renne (1970) encontró que las personas que desempeñan ocupaciones de poco prestigio, con bajos ingresos y bajo nivel de escolaridad estaban más insatisfechas con sus matrimonios. Al respecto, Campbell, Converse y Rodgers (1976) sugieren que una educación profesional tiene efectos más importantes en la felicidad conyugal que los incrementos en escolaridad a niveles más bajos. Estos autores encontraron que las esposas menos satisfechas son las profesionistas y proponen que la felicidad varía directamente con el nivel de escolaridad, mientras que la satisfacción marital lo hace inversa-

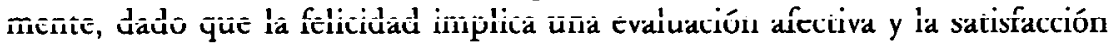
es de orden cognoscitivo.

Una última variable que se ha considerado en el presente estudio se refiere al número de hijos.

Desde Lang (1932) y más tarde Reed (1948) y Feldman (1964), se ha visto que los hijos afectan la interacción marital de manera tal que, existe una relación negativa entre número de hijos, satisfacción marital y ajuste marital. Glenn y Weaver (1978) encontraron esta misma relación entre tener hijos pequeños y satisfacción marital.

Renne (1970) encontró que parejas que se encuentran en el proceso de crianza están menos satisfechas en su relación marital que aquellas que tienen hijos adultos y viven fuera del hogar.

\section{Instrumento}

La satisfacción marital se definió como el grado de favorabilidad (actitud) hacia aspectos del cónyuge y de la interacción conyugal.

La Escala de Satisfacción Marital (Pick de Weiss y Andrade Palos, sometida a publicación) está compuesta por tres factores, a saber: satisfacción con los aspectos emocionales del cónyuge, que se refiere a la satisfacción que un cónyuge tiene con respecto a las reacciones emocionales de su pareja, satisfacción con 
la interacción conyugal, la cual trata con la satisfacción que un cónyuge tiene en lo que se refiere a la relación que lleva con su pareja y satisfacción con aspectos estructurales que mide la satisfacción que un cónyuge expresa de la forma de organización y de establecimiento y cumplimiento de reglas de su pareja. La consideración interna de cada subescala es alta (entre $\alpha=.81$ y $\alpha=.90$ ).

Las opciones de respuesta son: me gusta como está pasando, me gustaría que pasara de forma algo diferente, me gustaría que pasara de manera muy diferente.

Con respecto a las medias obtenidas para las subescalas de satisfacción con la interacción marital, con aspectos emocionales del cónyuge y con aspectos organizacionales y estructurales del cónyuge, fueron $\overline{\mathrm{X}}=15.2$ (rango posible 10-30), $\bar{X}=8.8$ (rango posible 5-12) y $\bar{X}=14.2$ (rango posible 9-27) respectivamente.

\section{Muestra}

La muestra estuvo constituida por 244 sujetos casados, 139 mujeres y 105 hombres. La edad promedio de los sujetos fue de 31.5 años, con un rango de 20 a 61 años. La escolaridad promedio fue de 11 años. El promedio de hijos, de 1.7. El $22 \%$ de la muestra no tenía hijos. El promedio de años de casados fue de 8.7 con un rango de 1 a 41 años. Los sujetos fueron seleccionados de ocho colonias de la Ciudad de México, elegidas al azar de todas las de nivel socioeconómico medio según el mapa de BIMSA (Buró de Investigación de Mercados, 1982). Dentro de cada colonia se tocaba en cada casa y se entrevistaban las personas casadas dispuestas a responder. Se reportaron 14 rechazos.

\section{Resultados}

Con el objeto de ver las diferencias en el rango de satisfacción marital en relación a cada una de las variables sociodemográficas bajo consideración, se aplicaron análisis de varianza de una entrada (one way).

Los grupos de cada variable se conformaron de la siguiente forma: sexo (masculino-femenino); edad (20 a 24 años, 25 a 26,27 a 29,30 a 32,33 a 39 y 40 ó más); escolaridad (primaria, secundaria, preparatoria, profesional incompleta, profesional completa o posgrado); número de hijos (ninguno, uno, dos, tres o más); número de años de casados (1 a 2, 3 a 4, 5 a 7, 8 a 15, 16 ó más). La conformación de los grupos se hizo con base en la frecuencia de las variables y considerando que fuera conceptualmente adecuada.

Para las variables que mostraron diferencias significativas, se aplicó la prueba post hoc de Scheffé con el fin de conocer en qué grupos estaban estas diferencias.

\section{Satisfacción con la interacción conyugal (SIC):}

Las variables: sexo, escolaridad, número de hijos y número de años de casados, mostraron diferencias significativas en la subescala de la interacción conyugal. Los grupos entre los que se encontraron las diferencias ( $p<.05$ en la prueba post hoc de Scheffé) son los siguientes: los hombres están más satisfechos con la interacción conyugal que las mujeres $(\overline{\mathrm{X}}=15.93$ vs $\overline{\mathrm{X}}=17.47)$. Puntales bajos significan mayor satisfacción. Las personas que tienen uno o dos años de casados están más satisfechas que las que tienen 16 años o más de casados $(\overline{\mathrm{X}}=15.27$ vs $\overline{\mathrm{X}}=19.50)$. 
La satisfacción es menor en personas con tres o más hijos que en aquellas que tienen uno, dos, o bien no tienen hijos $(\overline{\mathrm{X}}=19.6$ vs $\overline{\mathrm{X}}=15.94, \overline{\mathrm{X}}=$ $16.05, \bar{X}=15.57$ respectivamente). $Y$ por último, las personas que tienen escolaridad de secundaria $(\overline{\mathrm{X}}=19.04)$ están menos satisfechas que las que tienen una profesión $(\overline{\mathrm{X}}=15.37)$ (Tabla 1$)$.

TABLA I

Relaciōn entre Satisfacción Marital y variables socio-demográficas

\begin{tabular}{|c|c|c|c|c|c|}
\hline $\begin{array}{l}\text { Variables } \\
\text { Demográfica }\end{array}$ & Escalas & SIM • & SEM * & SES * & $\mathbf{N}$ \\
\hline Sexo & $\begin{array}{l}\text { Masculino } \\
\text { Femenino }\end{array}$ & $\begin{array}{l}15.93 \\
17.47\end{array}$ & $\begin{array}{l}8.66 \\
8.88\end{array}$ & $\begin{array}{l}11.83 \\
13.10\end{array}$ & $\begin{array}{l}105 \\
137\end{array}$ \\
\hline $\begin{array}{l}\text { Número } \\
\text { de } \\
\text { años de } \\
\text { casados }\end{array}$ & $\begin{array}{c}1-2 \\
3-4 \\
5-7 \\
8-15 \\
16 \text { ó más }\end{array}$ & $\begin{array}{l}15.27 \\
15.81 \\
17.77 \\
16.53 \\
19.50\end{array}$ & $\begin{array}{l}8.25 \\
8.60 \\
8.95 \\
8.66 \\
9.88\end{array}$ & $\begin{array}{l}11.71 \\
12.46 \\
12.95 \\
12.42 \\
13.57\end{array}$ & $\begin{array}{l}52 \\
48 \\
44 \\
53 \\
42\end{array}$ \\
\hline Edad & $\begin{array}{c}20-24 \\
25-26 \\
27-29 \\
30-32 \\
33-39 \\
40 \text { ó más }\end{array}$ & $\begin{array}{l}15.74 \\
16.48 \\
17.04 \\
15.76 \\
17.06 \\
18.84\end{array}$ & $\begin{array}{l}8.38 \\
8.97 \\
8.54 \\
8.34 \\
8.86 \\
9.63\end{array}$ & $\begin{array}{l}11.69 \\
12.97 \\
12.95 \\
11.84 \\
12.51 \\
13.42\end{array}$ & $\begin{array}{l}39 \\
41 \\
44 \\
38 \\
43 \\
38\end{array}$ \\
\hline Escolaridad & $\begin{array}{c}\text { Primaria } \\
\text { Secundaria } \\
\text { Preparatoria } \\
\text { Profesional } \\
\text { Inc. } \\
\end{array}$ & $\begin{array}{l}18.00 \\
19.04 \\
16.83 \\
15.37\end{array}$ & $\begin{array}{l}9.27 \\
9.35 \\
8.91 \\
8.05\end{array}$ & $\begin{array}{l}13.07 \\
13.35 \\
12.94 \\
11.70\end{array}$ & $\begin{array}{l}41 \\
46 \\
47 \\
40\end{array}$ \\
\hline $\begin{array}{c}\text { Número } \\
\text { de } \\
\text { Hijos }\end{array}$ & $\begin{array}{l}\text { Ninguno } \\
\text { Uno } \\
\text { Dos } \\
\text { Tres o más }\end{array}$ & $\begin{array}{l}15.57 \\
15.94 \\
16.05 \\
19.60\end{array}$ & $\begin{array}{l}8.58 \\
8.76 \\
8.07 \\
9.68\end{array}$ & $\begin{array}{l}11.49 \\
11.44 \\
12.35 \\
13.87\end{array}$ & $\begin{array}{l}55 \\
72 \\
57 \\
60\end{array}$ \\
\hline
\end{tabular}

* Puntajes bajos significan mayor satisfacción

Respecto a las diferencias entre hombres y mujeres; se encontró que los hombres están más satisfechos que las mujeres en lo que se refiere a la interacción marital y los aspectos estructurales del cónyuge, lo cual confirma algunos hallazgos previos (e.g. Atkinson, 1980; Bernard, 1972; Campbell, Converse y Rodgets, 1976; Rhyne, 1981).

Parece ser que resultados contradictorios encontrados previamente en la satisfacción marital entre los sexos se pueden explicar en términos de la interacción y aspectos estructurales y emocionales del cónyuge, siendo que las diferencias entre los sexos se encuentran en las dos primeras y no en la tercera.

Los resultados encontrados con respecto al número de años de casados contradicen estudios anteriores que apuntan hacia una relación en forma de «U» (Burr, 1970; Rollins y Cannon, 1974; Rollins y Feldman, 1970); y apoyan el modelo lineal de decremento de la satisfacción marital (Pineo, 1961; Swensen, Eskew y Kohlepp, 1981). Sin embargo, cabe destacar que las diferencias en el ciclo 
marital se encontraron solamente en aspectos de la interacción marital y no en aspectos emocionales y estructurales. El que no se hayan encontrado diferencias en los aspectos emocionales y estructurales se podría deber a que éstas no sufren tanto cambio a través del ciclo vital, ya que son aspectos de personalidad más que situacionales, mientras que la interacción marital es un aspecto que puede verse más afectado por causas externas a las características propias de cada miembro de la pareja. Con respecto a este punto resulta necesario llevar a cabo estudios que centren su esfuerzo en el análisis de la velocidad e intensidad con la que se modifica la satisfacción marital en relación a variables extrínsecas de la pareja, tales como: número de hijos, familia extensa, amigos, cambios socioeconómicos y de salud.

Con respecto a las diferencias en escolaridad entre sujetos con nivel medio y alto, los resultados apoyan lo encontrado por Campbell, Converse y Rodgers (1976) quienes afirman que la satisfacción conyugal se ve más afectada por niveles de educación altos que por incrementos de escolaridad a niveles bajos.

Asimismo el que la diferencia en satisfacción con la interacción marital en escolaridad se da entre el nivel medio y alto se puede explicar de acuerdo a resultados semejantes obtenidos anteriormente (Arias Galicia, 1985) que explican sus resultados en base a que en los niveles medios hay más posibilidad de comparación con los niveles altos y que la insatisfacción marital en este caso es reflejo de una insatisfacción general. No se da en los aspectos estructurales y emocionales dado que posiblemente están menos sujetos a cambios situacionales.

En relación al número de hijos se observa que el tener tres o más hijos afecta la satisfacción marital en las tres subescalas. El menor grado de satisfacción con la interacción se encuentra en personas con tres o más hijos, lo cual puede ser explicado en términos de las presiones y la dimensión de posibilidades de interacción de la pareja cuando hay que cuidar y criar muchos hijos (Glenn y Weaver, 1978). El cambio en satisfacción con aspectos emocionales con respecto al número de hijos parece estar de dos a tres o más, lo cual se puede explicar en los mismos términos que el hallazgo anterior. Igualmente para los aspectos estructurales parece ser que a pesar que la diferencia entre ninguno, uno y dos hijos podría ser importante, la diferencia estadísticamente significativa está entre parejas sin hijos y con tres o más. La organización, la dedicación, el tiempo, las reglas que requieren familias con muchos hijos es muy diferente a la que se necesita cuando la pareja vive sola y no tiene que preocuparse por el desarrollo y bienestar de los niños (Glenn y Weaver, 1978).

El presente estudio representa un primer paso en la cultura mexicana hacia la comprensión de la satisfacción marital y las diferencias entre grupos. El siguiente paso es el desarrollo de estudios longitudinales que permitan explicar con bases empíricas cada uno de los puntos contemplados en la presente investigación.

\section{Referencias}


BALTES, P.B. (1968). \&Longitudinal and cross-sectional sequence in the study of age and generational effectss, Human Development, 11(3), 145-171.

BERGER, P.L. y KeIINER, H. (1970). \&Marrigage and the Construction of Realitys en H.P. Dreitzel (ed.) Recent Sociology, No. 2, Londres: McMillan.

BERNARD. J. (1972). The Future of Marriage, Nueva York: Bantam Books.

Bioon. R.O. y Wolfe, D.M. (1960). Husband and Wives, the Dynamics of Married Living, Nueva York: The Free Press.

BURR, W.R. (1970). \& Satisfaction with various aspects of marriage over the life cycle a random middle class samplex, Joumal of Marriage and the Family, 32, 29-37.

CAMPBELL. A., CONVERSE, P.E. y RODGERS, W.L. (1976). The Quality of American Life, Nueva York: Russell Sage Foundation.

Chadwick, B.A., AlbreCht. S.L. y Kunz, P.R. (1976). «Marital and Family Role Satisfaction», Journal of Marriage and the Family, 431-440.

DuvaL, E. (1971). Family Development, Philadelphia: Lippincott.

Feldman, H. (F1964). Development of the Husband-Wife Relationship, a research Report, Ithaca, N.Y.; Cornell University (mimeógrafo).

GLENN, N.A. y WEAVER, C.N. (1978). A multivariate, multisurvey study of marital happiness, Journal of Marriage and the Family, 269-282.

HiCKS, M.W. y PLATT, M. (1970). Marital Happiness and Stability: A review of the Research in the Sixtiess, Joumal of Marriage and the Family, 533-574.

LANG, R.O. (1932). A study of the degree of happiness or unhappiness in Marriage, tesis de maestría, Universidad de Chicago.

LAWS. J.L. (1971). A feminist review of the marital adjustment literature: the rape of the locke, Jour nal of Marriage and the Family, 33, 483-516.

LOCKE, H.J. y WALACE, K.M. (1959). \&Short marital-adjustment and prediction tests: their reliability and validitys, Mamiage and Family Living, 251-255.

NoCK, S.L. (1979). The Family Life Cycle: Empirical or Conceptual tool? , Joumal of Marriage and the Family, 15-26.

PICK DE WeISS, S. y ANDRAde PALOS, P. (sometido a publicación). Desarrollo y validación de la Escala de Satisfacción Marital.

PINEO, P.C. (1961). sDisenchantment in the later years of marriages Jourmal of Marriage and the Family, 941-955.

REED, R.B. (1948). Social and Psychological Factors Affecting Fertility, Nueva York: Milbank Memorial Fund.

RENNE, K. (1970). CCorrelates of dissatisfaction in marriages, Joumal of Marriage and the Family, 32-54-56.

RHYNE. D. (1981). *Bases of marital satisfaction among men and women,, Jourmal of Marriage and the Family. 941-955.

ROACH, A.J., FrAZIER, L.P. y Bowden, S.R. (1981). «The Marital Satisfaction Scale: development of a measure for intervention Researchs, Journal of Marriage and the Family, 537.546.

ROIINS, B.C. y CANNON, K.L. (1974). Marital satisfaction over the family life cycle: A reevaluations, Joumal of Marriage and the Family, 36, 217-282.

RoLuINS, B.C. y FELDMAN, H. (1970). eMarital satisfaction over the life cycles, Joumal of Marriage and the Family, 32, 20-27.

ROLINS, B.C. y GALUGAN, R. (1978). The developing child and marital satisfaction of parentss en R. Lenner y G. Spanier (eds.) Children's influence on Marital and Family Interaction: A Life Span Perspective, Nueva York: Academic Press.

SPANIER, G.B. (1976). «Measuring dyadic adjustment: new scales for assesing the quality of marriage and similar dyadss, Joumal of Marriage and the Family, 38, 15-28.

SPANIER, G.B., LEWIS. R.A. y COLE, C.L. (1975). a Marital adjustment over the family life cycle: the issue of curvilinearity, Joumal of Marriage and the Family, 37, 263-275.

SPANIER, G.B., SAUER, W. y LARZCLERE, R. (1979). aAn empirical evaluation of the family life cyclen, Joumal of Marriage and the Family, 41, 27-38.

SWENSEN. C.H., ESKEW, R.W. y KOHLHEPP (1981). stage of Family Life Cycle, Ego Development and the Marriage Relationship», Joumal of Marriage and the Family, 841-853. 